\title{
Probing the sensitivity of orientational ordering as a way towards absolute enantiorecognition: Helical-particle solutes in helical-particle nematic solvents
}

\author{
Jaime Gutiérrez Bojart \\ Departamento de Física Teórica de la Materia Condensada, Universidad Autónoma de Madrid, Ciudad Universitaria de Cantoblanco, \\ E-28049 Madrid, Spain \\ Christian D'Urso and Giorgio Celebre \\ Dipartimento di Chimica e Tecnologie Chimiche, Università della Calabria, via Pietro Bucci, I-87036 Arcavacata di Rende (CS), Italy \\ Giorgio Cinacchi* \\ Departamento de Física Teórica de la Materia Condensada, Instituto de Física de la Materia Condensada (IFIMAC) \\ and Instituto de Ciencias de Materiales "Nicolás Cabrera”, Universidad Autónoma de Madrid, Ciudad Universitaria de Cantoblanco, \\ E-28049 Madrid, Spain
}

(Received 19 June 2018; published 30 October 2018)

\begin{abstract}
To inquire into whether two enantiomers can be absolutely distinguished on the sole basis of their different degrees of orientational ordering when dissolved in a chiral nematic liquid crystal, several enantiomeric pairs of hard helical particles are dissolved in a cholesteric (nematic) liquid crystal made of slender hard helical particles as well as in a screwlike nematic liquid crystal made of tortuous hard helical particles. While in the former ordinary chiral nematic solvent their nematic order parameters are (almost) coincident, in the latter new chiral nematic solvent the two enantiomeric solutes not only usually have more appreciably different nematic order parameters, but also always have significantly different screwlike order parameters. If also the latter orientational order parameter could be measurable in real experiments, it would constitute an additional decisive piece of information on the way to absolutely distinguishing two enantiomers on the sole basis of their different degrees of orientational order when dissolved in a chiral nematic liquid crystal that is in the apter screwlike nematic phase. Even in that event, however, the general absence of regularity and systematicity in the trend of the orientational order parameters, already manifest in these elementary hard helical-particle binary systems, would make this coveted achievement experimentally arduous without the assistance of very accurate and precise theoretical calculations.
\end{abstract}

DOI: 10.1103/PhysRevE.98.042704

Within the very broad subject of chirality [1], a basic problem in (soft) condensed matter science [2], with its potentially far-reaching biological, medical, and pharmaceutical implications, is to absolutely distinguish between, i.e., to assign the absolute configuration of, two enantiomers $[3,4]$.

In theory, one possible procedure to achieve this objective is to dissolve the two enantiomeric (molecular or colloidal) particles in a chiral nematic liquid crystal [5]. Since the interactions between the solute and the solvent particles are diastereoisomericlike [3,4] and the solvent is orientationally ordered, the two enantiomeric solute particles, apart from a different solubility, will also have a different degree of orientational ordering and thence exhibit different orientational properties. The subsequent measurement and suitable analysis of these properties might enable the enantiomorphicity of the two solute particles to be retrieved.

In practice, the formidable difficulty that one is often faced with when addressing (molecular or colloidal particle) chirality is that too minute and subtle are the differences between the (interactions involving the) two enantiomeric particles to

\footnotetext{
*giorgio.cinacchi@uam.es
}

be unambiguously detectable and discernible: "They are easily washed out in any, even mildly rippled, sea": In retrospect, it is not that surprising that the first enantiomeric resolution (separation) could be of a chiral molecular substance in its crystalline (positionally and orientationally ordered) rather than fluid (at most only partially ordered) phase [6] as well as that the first experimental technique to assign the absolute configuration of a chiral molecule had been anomalous x-ray diffraction applied to a sample of them in the crystalline phase [7].

The common chiral nematic phase is the cholesteric $\left(N_{c}^{*}\right)$ phase [5]. In this phase, that may be formed in any single- or pluricomponent nonracemic system of chiral particles and potentially nematogenic particles, the local nematic director $(\hat{n})$ rotates in a (circular) helical fashion around a perpendicular helical axis $(\hat{\mathbf{h}})$ with a certain half-pitch $\mathcal{P} / 2$ usually orders of magnitude larger than particle dimensions and sensitively depending on the particle chirality and the (thermodynamic) state of the system [8-12]. To a very good approximation, the $N_{c}^{*}$ phase is locally equivalent to the corresponding uniaxial nematic $\left(N^{*}\right)$ phase obtainable as $\mathcal{P} \rightarrow \infty$ [13].

Out of the experimental techniques that can participate in that possible procedure, one is nuclear magnetic resonance 


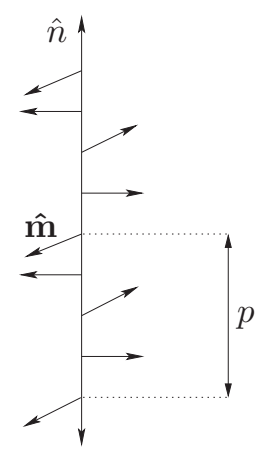

FIG. 1. Schematic of the secondary director $\hat{\mathbf{m}}$ perpendicularly twisting around the primary director $\hat{n}$ with pitch $p$ coincident in sense and value with that of the helical particles constituting the system in the screwlike nematic phase.

(NMR) spectroscopy [14]. Since the first report on the NMR spectrum of benzene as a solute in an ordinary nematic solvent [15], the NMR spectroscopy in liquid-crystal solvents (LXNMR) [16] has proved, via the measurement of the NMR spectrum and its analysis to yield residual dipolar couplings (RDCs), to accurately determine, apart from the structure, the second-order orientational (nematic) order parameter of rigid small solute molecules $[17,18]$.

Current LX-NMR experiments commonly employ, as a chiral nematic solvent, a lyotropic solution of a chiral polypeptide, such as poly- $\gamma$-benzyl-L-glutamate (PBLG), in a suitable organic liquid. The $10-\mu \mathrm{m}$-long- $\mathcal{P} N_{c}^{*}$ phase that such a system forms $[19,20]$ is weakly orienting and when immersed in a magnetic field ordinarily transforms by the cholesteric helix unwinding into a $N^{*}$ phase [21].

Though the weakly orienting character of such a liquidcrystal solvent is welcome as allowing for analyzable nearly first-order (quasi-isotropic) (pro)chiral solute NMR spectra that, thanks to the enantiomeric excess of the solvent polymeric particles and therefore the diastereoisomericlike nature of the resultant solute-solvent interactions, often show well-separated NMR signals [22], it however exacerbates the already possible inadequacy of its intrinsic uniaxial nematicity [23] to unambiguously distinguish the two enantiomers.

In the absence of a meticulously reliable theoretical prediction of the solute degree of orientational ordering in a (chiral) nematic solvent, the intrinsic uniaxial nematicity [23] along with the incapability by the solvent of sufficiently strongly orienting the solutes would have already been sufficient reasons for sceptically regarding the recent claims [24,25] of having assigned the absolute configuration of the two enantiomers via the measurement of the RDCs in LX-NMR experiments using as a liquid-crystal solvent a PBLG or similar lyotropic solution in addition to the serious specific concerns explicitly raised [26] on the validity of those claims.

Recently, evidence has been provided for the existence of another distinct chiral nematic phase, denoted the screwlike nematic $\left(N_{s}^{*}\right)$ phase, in (enantiopure) systems of helical particles [27-29]. In this new chiral nematic phase, the primary nematic director $\hat{n}$ remains fixed, and it is a secondary nematic director $\hat{\mathbf{m}}$ that rotates around it in a (circular) helical fashion $(\hat{\mathbf{h}} \| \hat{n})$ with a pitch coincident in sense and value with the pitch of the constituent helical particles (Fig. 1). Different than the $N_{c}^{*}\left(N^{*}\right)$ phase, the $N_{s}^{*}$ phase is locally strongly biaxial as well as polar.

For this reason, a solvent in the $N_{s}^{*}$ phase may be better suited than one in the $N_{c}^{*}\left(N^{*}\right)$ phase in amplifying the difference between the two enantiomeric solute particle orientational order parameters thus easing if not at all enabling their distinguishability. This work wishes to explore this possibility. For this purpose, model binary systems of hard helical particles are considered and investigated via numerical simulation.

Each helical particle is (for simplicity) composed of a number $n_{s}$ of hard spheres of diameter $D$ whose centers are equidistantly and rigidly arranged along a helical chord of length $L$, radius $r$, and pitch $p$ as in previous works [29]. The mechanical state of any such helical particle is defined by the centroid $\mathbf{r}_{\circ}$ and the left- or right-handed triad of mutually perpendicularly unit vectors $\{\hat{\mathbf{u}}, \hat{\mathbf{v}}, \hat{\mathbf{w}}\}$ with $\hat{\mathbf{u}}$ along the particle helical axis and $\hat{\mathbf{w}}$ along the particle $C_{2}$ axis (Fig. 2). By knowing the parameters $L, r$, and $p$ of a helical particle together with its $\mathbf{r}_{\circ}$ and $\{\hat{\mathbf{u}}, \hat{\mathbf{v}}, \hat{\mathbf{w}}\}$, the position $\mathbf{q}_{i}$ of the $i$ th, $1 \leqslant i \leqslant n_{s}$, hard-sphere center can be retrieved

$$
\mathbf{q}_{i}=\mathbf{r}_{\circ i}+r \cos \left(2 \pi t_{i}\right) \hat{\mathbf{w}}+r \sin \left(2 \pi t_{i}\right) \hat{\mathbf{v}}+p t_{i} \hat{\mathbf{u}},
$$

with $t_{i}=\left[-\frac{1}{2}\left(n_{s}-1\right)+i-1\right] \Delta t$ and $\Delta t$ such that

$\frac{L}{n_{s}-1}=2 \pi \Delta t \sqrt{r^{2}+\left(\frac{p}{2 \pi}\right)^{2}}$. For any examined binary system, the vast majority of its constituent particles, i.e., the solvent particles, are right-handed $(\Delta, \mathrm{D}$ or plus, P) helical particles always with $L=10 D$ and composed of $n_{s}=15$ (partially overlapping) hard spheres. In this work, two specific types of helical solvent particles are considered. In one case, they have $r / D=0.2$ and $p / D=9.92$, while in the other case, they have $r / D=0.4$ and $p / D=4$. The former helical particles are nearly straight rodlike and able to form the $N_{c}^{*}\left(N^{*}\right)$ phase [29]; the latter helical particles are considerably curlier and able to form the $N_{s}^{*}$ phase [29] (Fig. 2). In these solvents, several different helical solute particles are dissolved. They are chosen to have $(r / D, p / D)=\{(0.2,9.92) ;(0.2,4) ;(0.4,4) ;(0.4 ; 2)\} \quad$ and composed of $n_{s}=2 k_{s}+1, k_{s} \in[1,2, \ldots, 7]$ (partially overlapping) hard spheres of diameter $D$ with their respective $L$ varying with $n_{s}$ such that $L=(5 / 7)\left(n_{s}-1\right) D$; they are either left $(\Lambda, L$ or minus, $M)$ or right handed except those with $n_{s}=3$ that clearly are achiral [30] (Fig. 2).

Binary systems of such hard helical particles were investigated via the Monte Carlo (MC) method in the isobaric (-isothermal) (NPT) statistical ensemble [31-33] with orthorhombic periodic boundary conditions. Configurations of $N \approx 500$ identical right-handed hard helical particles either with $(r / D, p / D)=(0.2,9.92)$ or with $(r / D, p / D)=$ $(0.4,4)$ coming from previous numerical simulations [29] were taken [34]. The configuration with straighter hard helical particles was obtained in the course of a production run at the state point in the $N_{c}^{*}\left(N^{*}\right)$ phase with $P D^{3} / k_{B} T=0.5$ and mean number density $\varrho D^{3}=0.044$ while that with the curlier hard helical particles was obtained in the course of a production run at the state point in the $N_{s}^{*}$ phase with $P D^{3} / k_{B} T=0.8$ and a mean number density $\varrho D^{3}=0.051$. For each of the examined solute-solvent cases, ten randomly chosen helical solvent particles of these configurations were replaced by helical solute particles taking due care that the 
(a)

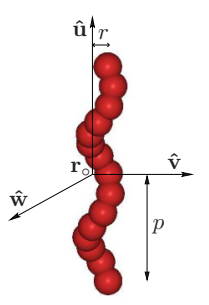

(b)

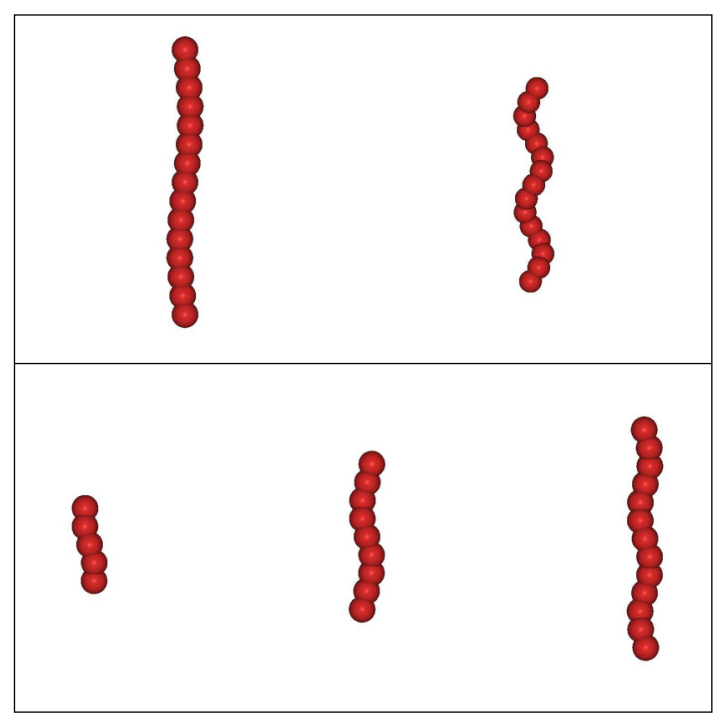

FIG. 2. (a) Image of a helical particle composed of 15 partially overlapping spherical beads of diameter $D$ whose centers are arranged along a helical chord of length $L$, radius $r$, and pitch $p$. The mechanical state of such a helical particle can be defined by specifying its frame of reference: the position of the center $\mathbf{r}_{\circ}$, and the orientation of the triad of mutually perpendicular unit vectors $\hat{\mathbf{u}}, \hat{\mathbf{v}}$, and $\hat{\mathbf{w}}$. The positions of the component spherical beads, when projected on the $(\hat{\mathbf{v}}, \hat{\mathbf{w}})$ plane, lie on a circumference with center $\mathbf{r}_{\circ}$ and radius $r$; the axis along $\hat{\mathbf{u}}$ is the one along which the helix unrolls with a pitch $p$. (b) Images of a few helical particles considered in the present work: In the top panel there are the two right-handed solvent particles, the one with $r / D=0.2$ and $p / D=9.92$ on the left and the one with $r / D=0.4$ and $p / D=4$ on the right; in the bottom panel there are three left-handed solute particles with $r / D=0.2$, $p / D=4$, and $n_{s}=5,9,13$.

latter did not overlap with any remaining helical solvent particle. From these 20 configurations thus generated, new MC$N P T$ calculations were initiated. These calculations were organized in cycles each of them consisting of $N$ attempts to translate a randomly selected particle, $N$ attempts to rotate a randomly selected particle around one randomly selected axis of the laboratory reference frame, $N$ attempts to rotate a randomly selected particle around its $\hat{\mathbf{u}}$, and one attempt to change the shape and size of the computational box by randomly selecting one of the three edges and changing its length. Once an equilibration run of 500k MC cycles had been accomplished, in the production run of twice as many MC cycles thousands of configurations were regularly saved and stored for the subsequent analysis.

This analysis primarily involved the calculation of the (Saupe) order matrix $(\mathcal{S})$ [36], the nematic order parameter of both the solute $(\sigma)$ and the solvent $(\Sigma)$, whose elements are defined as

$$
\mathcal{S}_{\alpha \beta}^{\iota}=\left\langle\frac{3}{2}(\hat{\boldsymbol{\alpha}} \cdot \hat{n})(\hat{\boldsymbol{\beta}} \cdot \hat{n})-\frac{1}{2} \delta_{\alpha \beta}\right\rangle,
$$

with $\iota=\sigma, \Sigma ; \hat{\boldsymbol{\alpha}}, \hat{\boldsymbol{\beta}}=\hat{\mathbf{u}}, \hat{\mathbf{v}}, \hat{\mathbf{w}} ; \delta_{\alpha, \beta}$ as the Krönecker symbol; 〈〉 signifying an arithmetic mean over particles and configurations. The analysis also involved the calculation of pair correlation functions, such as $g^{\ell, \kappa}(r)$ and $g_{1, \hat{\mathbf{w}}}^{\ell, \kappa}\left(r_{\|}\right)$. The positional pair correlation function $g^{l, \kappa}(r)$ is defined as

$$
g^{\iota, \kappa}(r)=\frac{1}{N_{\iota}}\left\langle\frac{1}{\varrho_{\kappa}} \sum_{i=1}^{N_{\iota}} \sum_{j=1 ; j \neq i}^{N_{\kappa}} \delta\left(r-r_{i j}\right)\right\rangle,
$$

with $N_{\iota}$ the number of particles of species $\iota, \varrho_{\kappa}$ the number density of species $\kappa$, the further specification $j \neq i$ applying if $\iota=\kappa, \delta()$ the usual $\delta$ function, $r_{i j}$ the modulus of the distance $\mathbf{r}_{i j}$ separating the centroid of particle $j$ from the centroid of particle $i$, and \langle\rangle signifying an arithmetic mean over the configurations. It is proportional to the conditional probability of finding the particle $j$ centroid in the spherical shell of radii $r$ and $r+d r$ centered on the particle $i$ centroid, and its form serves to confirm the positionally disordered (nematic) character of the investigated system. The orientational pair correlation function $g_{1, \hat{\mathrm{w}}}^{\ell, \kappa}\left(r_{\|}\right)$is defined as [29]

$$
g_{1, \hat{\mathbf{w}}}^{\iota, \kappa}\left(r_{\|}\right)=\left\langle\frac{\sum_{i=1}^{N_{\iota}} \sum_{j=1 ; j \neq i}^{N_{\kappa}}\left(\hat{\mathbf{w}}_{i} \cdot \hat{\mathbf{w}}_{j}\right) \delta\left(r_{\|}-\mathbf{r}_{i j} \cdot \hat{n}\right)}{\sum_{i=1}^{N_{\iota}} \sum_{j=1 ; j \neq i}^{N_{\kappa}} \delta\left(r_{\|}-\mathbf{r}_{i j} \cdot \hat{n}\right)}\right\rangle .
$$

It informs on the degree of correlation between the unit vectors $\hat{\mathbf{w}}$ of two helical particles as a function of the projection of the distance between their centroids along $\hat{n}$. Thus, $g_{1, \hat{\mathrm{w}}}^{\Sigma, \Sigma}\left(r_{\|}\right)$distinguishes between the $N_{c}^{*}\left(N^{*}\right)$ and the $N_{s}^{*}$ phases: It is vanishingly flat in the former case and cosinusoidal in the latter case with the amplitude of the cosine defining the screwlike order parameter [29]; furthermore, in case the $\Sigma$ screwlike orientational ordering is present, $g_{1, \hat{\mathrm{w}}}^{\sigma, \Sigma}\left(r_{\|}\right)$reveals the propensity that the $\sigma$ helical particles have to conform or not to that special orientational ordering that the $\Sigma$ helical particles have established. The calculation of both $g^{l, \kappa}(r)$ and $g_{1, \hat{\mathrm{w}}}^{\sigma, \Sigma}\left(r_{\|}\right)$was carried out by approximating as usual the $\delta$ function with a square function of unit height and finite width which, in the present work, was $0.01 D$.

Figure 3 reports the values of $\mathcal{S}_{\hat{\text { ûu}}}^{\sigma}$ for the four types of hard helical-particle solutes in the solvents made of hard $(0.2,9.92)$ or $(0.4,4)$-helical particles while the Supplemental Material reports their full (Saupe) order matrices [37]. The observation of these results may lead to a couple of inferences. That a chiral nematic solvent in the $N_{s}^{*}$ phase be a necessary condition for any hope of clearly distinguishing two enantiomers on the sole basis of a different degree of orientational ordering to survive [cf., in Fig. 3, the left panels, referring to a solvent made of hard $(0.2,9.92)$-helical particles in the $N_{c}^{*}\left(N^{*}\right)$ phase with the right panels, referring to a solvent made of hard $(0.4,4)$-helical particles in the $N_{s}^{*}$ phase]. This hope can materialize if the $\sigma$ helical particle is: (I) sufficiently in tune with the $\Sigma$ helical particle, i.e., it has very similar helical parameters $r$ and, particularly, $p$; (II) sufficiently elongated, so as to acquire a good degree of orientational ordering. In this 

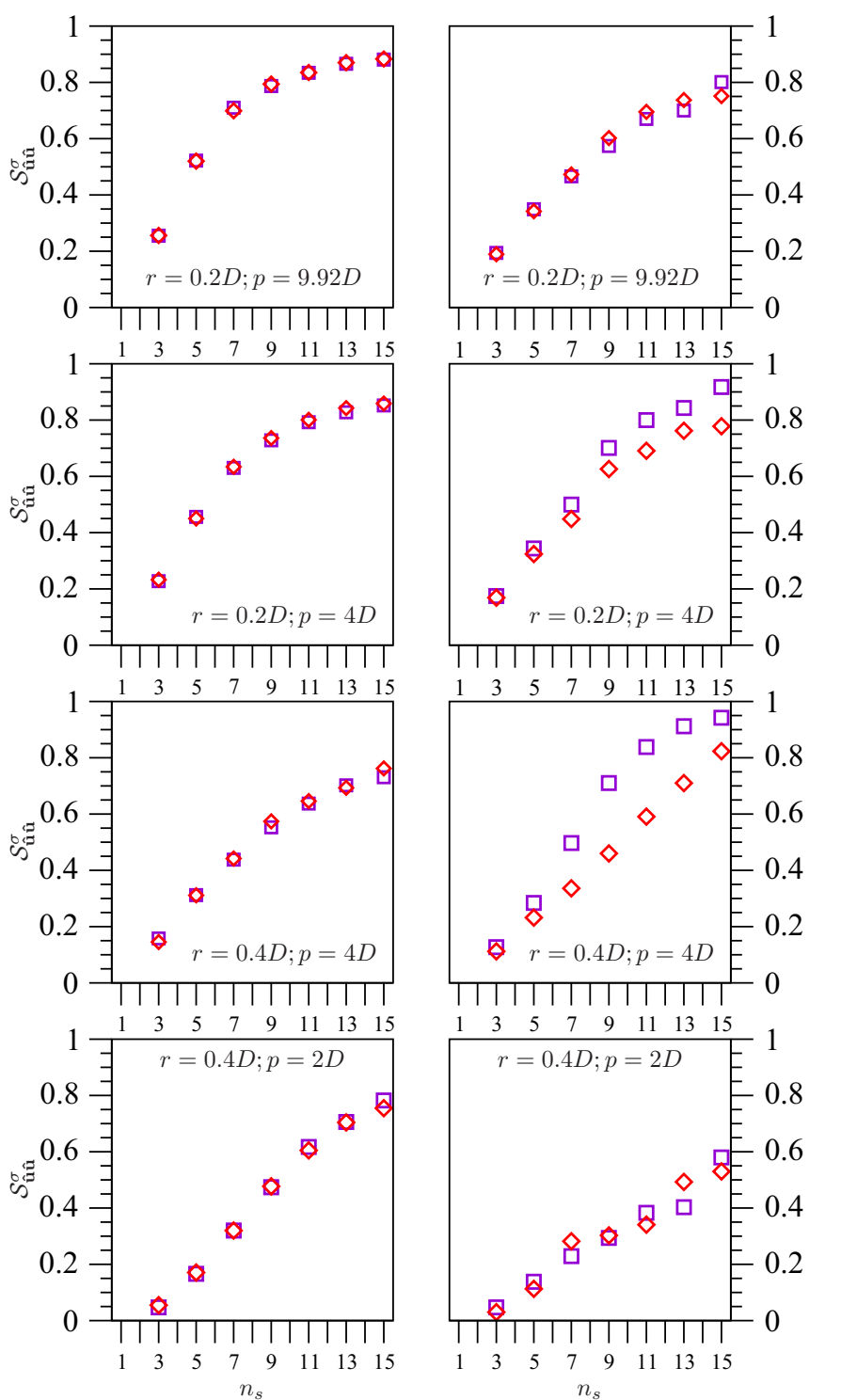

FIG. 3. The (Saupe) order matrix element $\mathcal{S}_{\hat{\text { ûu }}}^{\sigma}$ of the hard helicalparticle solutes both left (red diamond) and right handed (violet square), whose specific helical parameters are indicated within the respective square panel, either in the cholesteric solvent made of right-handed hard helical particles with $r=0.2 D ; p=9.92 D$ (left panels) or in the screwlike nematic solvent made of right-handed hard helical particles with $r=0.4 D ; p=4 D$ (right panels) as a function of the number of the constituent hard spheres $n_{s}$.

way, the difference between the orientational order parameters of the left- and right-handed $\sigma$ helical particles becomes more appreciable and overcomes the statistical error [cf., in Fig. 3, the two middle panels and the bottom panel on the right, referring to $\sigma(0.2,4)$ - or $(0.4,4)$ - or $(0.4,2)$-helical particles]. The orientational pair correlation functions $g_{1, \hat{\mathrm{w}}}^{l, \kappa}\left(r_{\|}\right)$confirm these expectations by revealing what are the (micro)structure features the results of Fig. 3 and the Supplemental Material [37] are rooted in.

Though those in the $N_{c}^{*}\left(N^{*}\right)$ phase invariably level to zero, the functions $g_{1, \hat{\mathrm{w}}}^{l, k}\left(r_{\|}\right)$in the $N_{s}^{*}$ phase are characteristically cosinusoidal (Fig. 4). Only the $\sigma$ right-handed hard

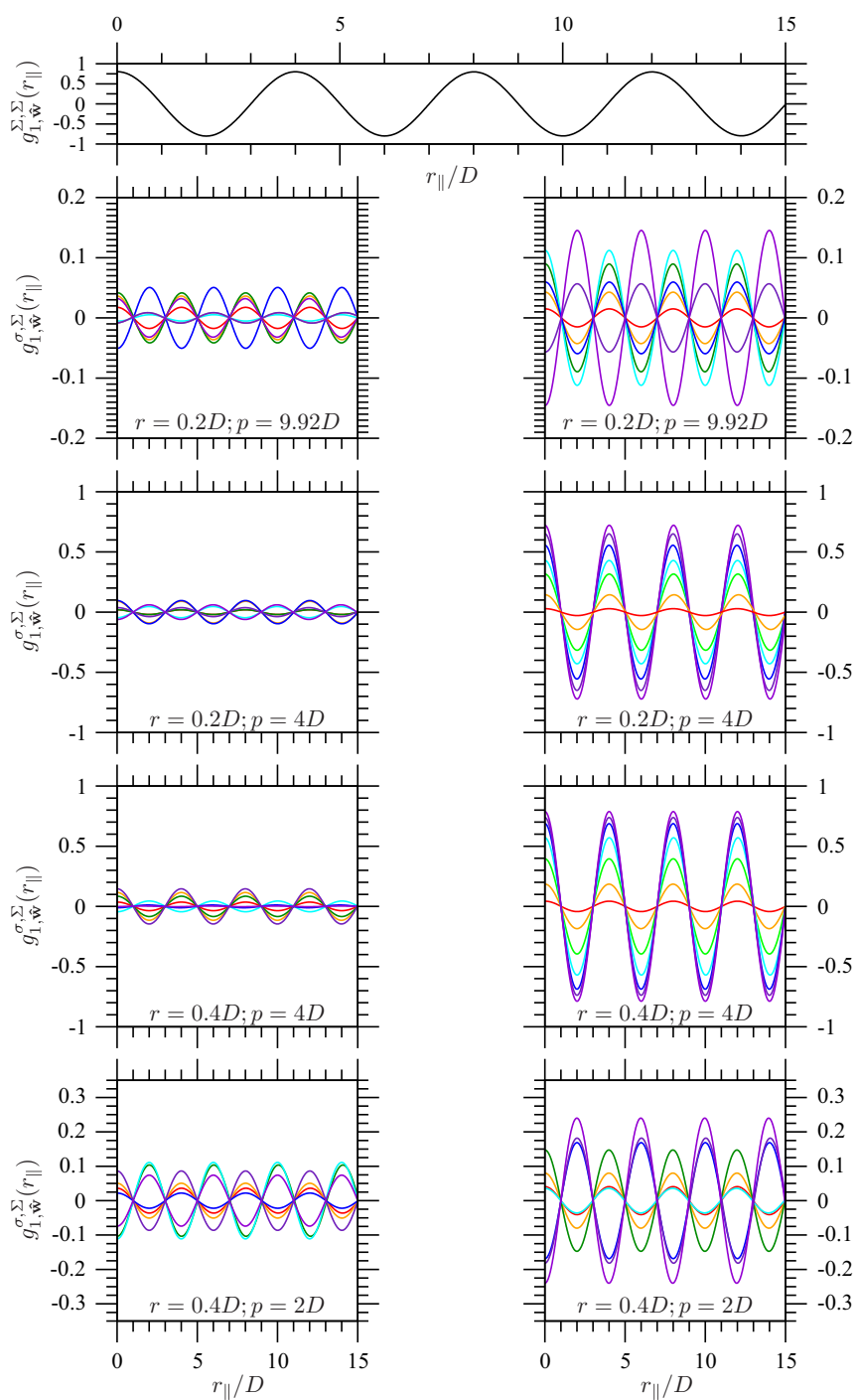

FIG. 4. The orientational pair correlation functions $g_{1, \hat{\mathrm{w}}}^{\sigma, \Sigma}\left(r_{\|}\right)$for the left- and right-handed hard helical solute particles, whose helical parameters are indicated within the respective square panel, in the screwlike nematic solvent made of right-handed hard helical particles with $r=0.4 D ; p=4 D$, whose orientational pair correlation function $g_{1, \hat{\mathbf{w}}}^{\Sigma, \Sigma}\left(r_{\|}\right)$is also shown in the topmost rectangular panel; in each square panel, results are shown for the several values of $n_{s}: 3$ (red); 5 (orange); 7 (green); 9 (cyan); 11 (blue); 13 (indigo); 15 (violet).

helical particles with the same value of $p=4 D$ are able to be syncorrelated with the $\Sigma$ right-handed hard helical particles; for these solute particles, the degree of screwlike orientational ordering regularly increases with $n_{s}$. Not only is the degree of screwlike orientational ordering of the $\sigma$ left-handed hard helical particles very small, but also irregularly varying with $n_{s}$, (interestingly) alternating cases in which the solute particles are syncorrelated with cases in which they are anticorrelated to the solvent particles. Such irregularity and smallness affect also the $\sigma$ right-handed hard helical particles with $p \neq 4 D$ though: in these cases, unambiguously distinguishing the two enantiomers on the sole basis of the nematic order parameters (Fig. 3) becomes impracticable. 
Keeping an eye on the real systems, this unfortunate situation would improve should an experimental technique be devised to accurately measure also the screwlike order parameter. This will constitute a piece of information in addition to the nematic order parameter. The conjunct pair of orientational order parameters will have a greater and more sensitive information content than the single nematic order parameter that will promote the distinguishability of two enantiomers.

For now, the achievement of measuring the screwlike order parameter, although difficult, may be previewed for colloidal systems: Shape-controllable colloidal helical particles with a micrometer-long $p$ are available that can form the $N_{s}^{*}$ phase [27]: By using such colloidal helical flagella, it may be that a real experimental study that parallels the present numerical simulation study is feasible. Whether that achievement could be previewed also for (in the present context, objectively more interesting) molecular systems will prerequire a major experimental effort towards the synthesis of suitably rigid helical (macro)molecules with a nanometer-long $p[38,39]$ and the characterization of their phase behavior to ascertain whether a $N_{s}^{*}$ phase is among the phases formed and, in this positive event, whether it can act as a chiral nematic solvent in LX-NMR experiments. In conjunction with the previous results [29], it is hoped that the present results, that demonstrate the greater suitability of the new $N_{s}^{*}$ phase than the ordinary $N_{c}^{*}\left(N^{*}\right)$ phase to be used as a chiral nematic solvent in LX-NMR experiments, will stimulate such experimental investigations.
The theoretical interpretation of the data of Fig. 3 should require classical density functional theory (DFT) calculations along the lines that were previously presented for rigid small solutes dissolved in smectic solvents [40]. Such a classical DFT needs to go beyond the second-virial approximation to properly deal with both the $N_{c}^{*}$ and especially the $N_{s}^{*}$ phases [41]. Even in the case of a pure system of hard helical particles that can form both types of chiral nematic phases, the implementation of such DFT calculations is yet to be accomplished but is planned. Such a DFT could then constitute the basis for a thermodynamic perturbative theoretical (TPT) treatment [42] aimed at predicting the degree of orientational ordering of a solute particle in either chiral nematic solvent.

Indeed, in view of the difficulty of absolutely distinguishing two enantiomers on the sole basis of their different degrees of orientational ordering, the achievement of such an objective using LX-NMR spectroscopy seems impracticable without the assistance of theoretical calculations aimed at reliably predicting the nematic order parameters of the two enantiomeric solute molecules. Irrespective of which theoretical method is chosen either DFT-TPT analytic or atomistic numerical simulational very high fidelity intermolecular interaction data will be required.

Support from the Università della Calabria and that from the Government of Spain under Grants No. FIS2013-47350C5-1-R, No. MDM-2014-0377, and No. FIS2017-86007-C3$1-\mathrm{P}$ is acknowledged.
[1] G. H. Wagnière, Chirality and the Universal Asymmetry: Reflections on Image and Mirror Image (Wiley-VCH, Weinheim, 2007).

[2] P. M. Chaikin and T. C. Lubensky, Principles of Condensed Matter Physics (Cambridge University Press, Cambridge, UK, 1995).

[3] G. Natta and M. Farina, Stereochimica: Molecole in 3D (Edizioni Scientifiche e Tecniche Mondadori, Milan, 1968); Stereochemistry (Harper \& Row, London, 1972).

[4] E. V. Anslyn and D. A. Dougherty, Modern Physical Organic Chemistry (Science University Books, Sausalito, CA, 2005).

[5] P. G. de Gennes and J. Prost, The Physics of Liquid Crystals (Clarendon, Oxford, 1993).

[6] L. Pasteur, C. R. Hebd. Séances Acad. Sci. 26, 535 (1948); Ann. Chim. Phys. 24, 442 (1848); H. D. Flack, Acta Crystallogr., Sect. A: Found. Crystallogr. 65, 371 (2009).

[7] J. M. Bijvoet, A. F. Peerdeman, and A. J. van Bommel, Nature (London) 168, 271 (1951); H. D. Flack, Acta Crystallogr., Sect. A: Found. Crystallogr. 39, 876 (1983).

[8] J. P. Straley, Phys. Rev. A 14, 1835 (1976).

[9] H. Toriumi, Y. Kusumi, I. Uematsu, and Y. Uematsu, Polymer J. 11, 863 (1979).

[10] J. Watanabe and T. Nagase, Macromolecules 21, 171 (1988).

[11] H. Stegemeyer, K. Siemensmeyer, W. Sucrow, and L. Appel, Z. Naturforsch. 44a, 1127 (1989).

[12] R. Memmer and F. Janssen, Liq. Cryst. 24, 805 (1998); R. Memmer and O. Fliegans, Mol. Phys. 101, 1829 (2003).

[13] It is agreed that the local equivalence between a $N_{c}^{*}$ phase and the corresponding $N^{*}$ phase be a very good approximation
[5,8]; nevertheless, its validity was explicitly tested on pure systems of certain hard helical particles [G. Muñoz Sánchez and G. Cinacchi (unpublished)].

[14] A. Abragam, The Principles of Nuclear Magnetism (Oxford University Press, Oxford, 1986).

[15] A. Saupe and G. Englert, Phys. Rev. Lett. 11, 462 (1963).

[16] J. W. Emsley and J. C. Lindon, NMR Spectroscopy Using Liquid Crystal Solvents (Pergamon, New York, 1975); Nuclear Magnetic Resonance of Liquid Crystals, edited by J. W. Emsley (Kluwer, Dordrecht, 1985); NMR of Ordered Liquids, edited by E. E. Burnell and C. A. de Lange (Kluwer, Dordrecht, 2003).

[17] E. E. Burnell and C. A. de Lange, Chem. Rev. 98, 2359 (1998); G. Celebre, G. De Luca, and M. Longeri, eMagRes 2, 335 (2013).

[18] One should note that the conditions of rigidity and smallness of the solute molecule are both necessary for the nematic order parameters to be accurate: The former prevents dealing with the complications due to the conformational degrees of freedom while the latter leads to a relatively simple NMR spectrum susceptible of being analyzed to give the nematic order parameters.

[19] A. Elliott and E. J. Ambrose, Discuss. Faraday Soc. 9, 246 (1950).

[20] C. Robinson, Trans. Faraday Soc. 52, 571 (1956); C. Robinson, J. C. Ward, and R. B. Beevers, Discuss. Faraday Soc. 25, 29 (1958); C. Robinson, Tetrahedron 13, 219 (1961); Mol. Cryst. 1, 467 (1966).

[21] P. G. de Gennes, Solid State Commun. 6, 163 (1968); G. Durand, L. Leger, F. Rondelez, and M. Veyssie, Phys. Rev. Lett. 22, 227 (1969). 
[22] B. Luy, J. Indian Inst. Sci. 90, 119 (2010); P. Lesot, Z. Luz, C. Aroulanda, and H. Zimmermann, Magn. Reson. Chem. 52, 581 (2014); P. Lesot, C. Aroulanda, H. Zimmermann, and Z. Luz, Chem. Soc. Rev. 44, 2330 (2015).

[23] The uniaxial character of such a nematic solvent is either effective and local when it is in the $N_{c}^{*}$ phase [13] out of the spectrometer or exact and global when it is in the $N^{*}$ phase in the spectrometer: A solute perceives this local, effective or exact, uniaxial nematicity.

[24] V. M. Marathias, G. J. Tawa, I. Goljer, and A. C. Bach, Chirality 19, 741 (2007).

[25] N. Nath and N. Suryaprakash, J. Phys. Chem. B 115, 6868 (2011).

[26] R. Berger, J. Courtieu, R. R. Gil, C. Griesinger, M. Köck, P. Lesot, B. Luy, D. Merlet, A. Navarro-Vázquez, M. Reggelin, U. M. Reinsheid, C. M. Thiele, and M. Zweckstetter, Angew. Chem. Int. Ed. 51, 8388 (2012).

[27] E. Barry, Z. Hensel, Z. Dogic, M. Shribak, and R. Oldenbourg, Phys. Rev. Lett. 96, 018305 (2006).

[28] F. Manna, V. Lorman, R. Podgornik, and B. Zeks, Phys. Rev. E 75, 030901(R) (2007).

[29] H. B. Kolli, E. Frezza, G. Cinacchi, A. Ferrarini, A. Giacometti, and T. S. Hudson, J. Chem. Phys. 140, 081101 (2014); H. B. Kolli, E. Frezza, G. Cinacchi, A. Ferrarini, A. Giacometti, T. S. Hudson, C. De Michele, and F. Sciortino, Soft Matter 10, 8171 (2014); H. B. Kolli, G. Cinacchi, A. Ferrarini, and A. Giacometti, Faraday Discuss. 186, 171 (2016); G. Cinacchi, A. M. Pintus, and A. Tani, J. Chem. Phys. 145, 134903 (2016); G. Cinacchi, A. Ferrarini, A. Giacometti, and H. B. Kolli, ibid. 147, 224903 (2017).

[30] The hard solute particles with $n_{s}=3$ can hardly be qualified as helical, and for them the triad $\{\hat{\mathbf{u}}, \hat{\mathbf{v}}, \hat{\mathbf{w}}\}$ hardly is an optimal particle-fixed frame of reference. [Helicity (thus chirality) and optimality of the triad $\{\hat{\mathbf{u}}, \hat{\mathbf{v}}, \hat{\mathbf{w}}\}$ would have been preserved if, maintaining the same length of the helical chord and the same diameter of the constituent hard spheres, the number of the latter had been larger.] Nevertheless, it was decided to maintain the same triad $\{\hat{\mathbf{u}}, \hat{\mathbf{v}}, \hat{\mathbf{w}}\}$ also for them.
[31] N. Metropolis, A. W. Rosenbluth, M. N. Rosenbluth, A. H. Teller, and E. Teller, J. Chem. Phys. 21, 1087 (1953).

[32] W. W. Wood, J. Chem. Phys. 48, 415 (1968); 52, 729 (1970).

[33] M. P. Allen and D. J. Tildesley, Computer Simulation of Liquids (Clarendon, Oxford, 1987); W. Krauth, Statistical Mechanics: Algorithms and Computations (Oxford University Press, Oxford, 2006).

[34] Numbers of particles of such an order of magnitude are typical in numerical simulations on (soft) condensed matter systems. They are sufficient to accurately calculate bulk properties of most three-dimensional fluid systems of hard particles [35].

[35] B. J. Alder and T. E. Wainwright, J. Chem. Phys. 33, 1439 (1960).

[36] A. Saupe, Angew. Chem. Int. Ed. 7, 97 (1968).

[37] See Supplemental Material at http://link.aps.org/supplemental/ 10.1103/PhysRevE.98.042704 for information. Being associated with the principal helical axis, the ûû element is considered as the principal element of $\mathcal{S}$ of a helical particle; for a certain consistency, it was taken as such also for the hard solute particles with $n_{s}=3$ [30].

[38] E. Yashima, K. Maeda, H. Iida, Y. Furusho, and K. Nagai, Chem. Rev. 109, 6102 (2009).

[39] M. Gingras, Chem. Soc. Rev. 42, 968 (2013); 42, 1007 (2013); 42, 1051 (2013).

[40] G. Cinacchi, Chem. Phys. Lett. 416, 238 (2005); G. Celebre, G. Cinacchi, and G. De Luca, J. Chem. Phys. 129, 094509 (2008); M. E. Di Pietro, G. Celebre, G. De Luca, and G. Cinacchi, Phys. Rev. E 84, 061703 (2011); M. E. Di Pietro, G. Celebre, G. De Luca, H. Zimmermann, and G. Cinacchi, Eur. Phys. J. E 35, 112 (2012).

[41] G. Cinacchi, A. Ferrarini, E. Frezza, A. Giacometti, H. B. Kolli, in Self-Assembling Materials: Theory and Simulation, edited by L. T. Yang (Wiley, Chichester, 2017), Chap. 3.

[42] C. G. Gray and K. E. Gubbins, Theory of Molecular Fluids Volume 1: Fundamentals (Oxford University Press, Oxford, 1984); C. G. Gray, K. E. Gubbins, and C. G. Joslin, Theory of Molecular Fluids Volume 2: Applications (Oxford University Press, Oxford, 2011). 